\title{
EVALUACIÓN AGRONÓMICA Y MEDIOAMBIENTAL DEL USO DE LA PAJA DE ARROZ COMO SUBSTRATO EN LA PRODUCCIÓN DE BIOGÁS
}

\author{
Alexis Ramón Zedeño Valmaseda ${ }^{[1]}$ \\ Bertha Carbonell Núñez ${ }^{[2]}$ \\ Yamisel Avalo López ${ }^{[3]}$
}

\section{Resumen}

Este trabajo constituye una revisión crítica sobre las afectaciones agronómicas y medioambientales que tiene la paja de arroz en la Empresa agroindustrial de granos Sur del Jíbaro, ubicado en la provincia de Sancti Spíritus, Cuba. Se hace una revisión literaria sobre el manejo de la paja de arroz en el mundo y en Cuba, así como las afectaciones agronómicas y medioambientales de la misma para los agroecosistemas; además, se hace un diagnóstico de las cantidades de paja producidas en dicha entidad en el 2008- 2013, así los principales destinos de éstas y una valoración de las afectaciones medioambientales debido a su manejo inadecuado. El trabajo hace una valoración de la factibilidad de utilización de dicha paja en biodigestores para la generación de energía.

Palabras clave: Paja de arroz; biodigestores; digestato.

\section{Summary}

This research is based on a critical analysis of the agronomic and environmental effects that the rice straw has on the agro-industrial grains company in South Jibaro, located in the province of Sancti Spiritus, Cuba. The methodology employed was a literature review on the management of rice straw in the world and particularly in Cuba, in order to determine the agricultural and environmental affectations to the agro-ecosystems. Besides, a diagnostic was made of the amount of straw produced in such entity during 2008-2013, as well as their main destinations and an evaluation of the feasibility of using the straw in bio-digesters for power generation.

Keywords: Rice straw; biodigesters; digestate.

[1] Lic. Director Centro Universitario de La Sierpe, Universidad de Sancti Spíritus "José Martí Pérez". E-mail: alexisz@uniss.edu.cu

[2] Licda. Docente de la Universidad de Sancti Spíritus "José Martí Pérez". E-mail: alexisz@uniss.edu.cu

[3] Licda. Docente de la Universidad de Sancti Spíritus "José Martí Pérez". E-mail: alexisz@uniss.edu.cu 


\section{Introducción}

Desde hace varios siglos la producción arrocera constituye una de las principales fuentes de alimentación del pueblo cubano formando parte de la canasta básica. Para la producción de este cultivo se han destinado numerosos recursos materiales dirigidos a obtener altos rendimientos, sin considerar las consecuencias de su aplicación sobre el ecosistema agrario a mediano y largo plazo.

Después de cada cosecha de arroz quedan en el campo restos de tallos y hojas a los que comúnmente se les denomina paja. Estos restos incorporados al suelo aportan elementos nutritivos que son asimilados por la planta durante el próximo período; sin embargo, este proceso de descomposición de la paja emite grandes volúmenes de gases contaminantes a la atmósfera lo cual afecta altamente el medio ambiente.

En el mundo se ha estudiado varias alternativas de uso para esta paja de arroz, algunas de las cuales consideran la extracción del campo de la misma. Esto implicaría que se estaría privando al cultivo de esa cantidad de nutrientes que antes se incorporaba a este.

Con este trabajo pretendemos evaluar las consecuencias medioambientales y agronómicas del uso de la paja de arroz como sustrato en la producción de biogás. No existe hoy en el mundo muchas experiencias que hablen sobre el tema y mucho menos en el país, por lo que se hace necesario el aporte de la ciencia para dilucidar tal disyuntiva que aportaría elementos teóricos para el manejo de la paja de arroz en los agroecosistemas arroceros cubanos con un enfoque medioambientalista.

El trabajo en si es una revisión crítica del tema a partir de las experiencias aportadas por diferentes autores en todo el mundo, a partir de las potencialidades para la producción de biogás a partir de la paja de arroz que tiene la empresa agroindustrial de granos Sur del Jíbaro ubicada en el municipio de La Sierpe, provincia de Sancti Spíritus, Cuba.

\section{Revisión de literatura}

El arroz es el segundo cereal de mayor producción en el mundo (le sigue al trigo), según explica la Organización de Naciones Unidas para la Agricultura y la Alimentación (FAO), en el mundo, hay 113 países que producen 400 millones de toneladas de grano de arroz; la mitad, sólo procede de China. Otros de los países productores son: España, Colombia, Perú y México.

Se estima que la producción mundial de arroz será de 760 millones de toneladas de arroz cáscara para el año 2025 (Gaddeet, et al., 2009), esto generaría aproximadamente 1140 millones de toneladas de residuos de cosecha. Estos están compuestos 
fundamentalmente por los tallos y las hojas de las plantas, conocido comúnmente como paja de arroz (Contreras, 2013).

La paja de arroz es uno de los subproductos más problemáticos de eliminar durante la cosecha del arroz. Ante la dificultad, elevado costo de su retirada y nulo aprovechamiento. Una de las prácticas más frecuentes por los agricultores es quemar la paja en el campo, lo que genera gran concentración de emisiones al aire de dióxido de carbono $\left(\mathrm{CO}_{2}\right)$, metano $\left(\mathrm{CH}_{4}\right)$ y otros en trazas como: óxido nitroso $\left(\mathrm{N}_{2} \mathrm{O}\right)$, monóxido de carbono (CO), hidrocarburos ( $\mathrm{HC}$ ), óxidos de nitrógeno (NOx) y óxidos de azufre (SOx), que provocan la contaminación del lugar y zonas aledañas, con las partículas y los gases resultantes de la combustión (Abril, et al., 2009).

Otra práctica común es la incorporación de la paja de arroz al suelo. Debido al proceso de degradación natural que ocurre bajo esas condiciones, existen emisiones, entre otros, de gas metano a la atmósfera (Watanable, et al., 1995). Además, esta práctica con el tiempo puede causar el deterioro de las condiciones del suelo en detrimento del rendimiento de la cosecha e incidir en el incremento de las enfermedades foliares (Zhang R. y Zhang Z., 1999).

$\mathrm{Al}$ quemarse la paja de arroz, los gases producidos son principalmente dióxido de carbono $\left(\mathrm{CO}_{2}\right)$, metano $\left(\mathrm{CH}_{4}\right)$ y otros en trazas como: óxido nitroso $\left(\mathrm{N}_{2} \mathrm{O}\right)$, monóxido de carbono (CO), hidrocarburos $(\mathrm{HC})$, óxidos de nitrógeno (NOxNox), óxidos de azufre (SOx) y agua.

$\mathrm{El}_{\mathrm{CO}}$ es el principal gas resultante. En un período de quema normal, todo el $\mathrm{CO}_{2}$ liberado pasará de nuevo a ser materia vegetal en el período de crecimiento del año próximo. Por tanto, la quema en los campos de residuos de cosechas no se considera una fuente neta de dióxido de carbono.

El metano ( $\left.\mathrm{CH}_{4}\right)$, es el segundo gas de efecto invernadero en importancia después del dióxido de carbono. Las fuentes emisoras de metano son en gran parte antropogénicas, como: las ciénagas, el cultivo de arroz, animales de granja, la quema de biomasa, entre otras. El metano contribuye a la formación de ozono troposférico, por tanto, su potencial como gas de efecto invernadero es superior al $\mathrm{CO}_{2}$. Se considera que el cultivo del arroz es el segundo gran responsable de las emisiones de metano. La combustión de la paja también genera metano, aunque muy poco en términos relativos (Fitzgerald, 200o), además de ser una fuente importante de dióxido de nitrógeno (NO2) a nivel local.

Las emisiones que emergen al quemar la paja de arroz son principalmente partículas de carbonilla en un $84 \%$ en peso (Dockery, et al., 1993). Además de su afectación a la salud (Kaufman, et al., 2002), reporta la capacidad de las PM2,5 y sus precursores para afectar el clima, ciclo hidrológico y visibilidad. 
Sin embargo, muchos agricultores de todo el mundo consideran que la quema de la paja de arroz, favorece la destrucción de las esporas de los hongos, como las de Pyricularia oryzae, bacterias, y semillas de malas hierbas y por otro lado facilita la reincorporación del Nitrógeno, Fósforo, Potasio y Sílice al suelo (Navarro, 2008).

Un equipo de investigadores del Instituto de Ciencia y Tecnología Animal (ICTA) de la Universidad Politécnica de Valencia en España ha trabajado el estudio comparativo sobre las emisiones de metano que genera el cultivo del arroz, en función de la técnica de gestión de la paja empleada: fangueo, quema o retirada. En el primero de los casos, del estudio se deriva que el fangueo genera mayores emisiones de metano que cualquier otra alternativa, con una emisión estacional de $418 \mathrm{~kg} / \mathrm{ha}$. Por debajo de ésta se encuentra la quema de la paja con una emisión de $239 \mathrm{~kg} / \mathrm{ha}$, mientras que lo que menos emisiones de metano genera es la retirada del campo de la paja (187 kg/ha).

La paja de arroz se retira de los campos en la India, Bangladesh y Nepal, donde se utiliza como combustible para cocinar, alimento de rumiantes, cama de animales, y otros procesos industriales. En estos casos no hay un retorno de nutrientes al suelo (Doberman \& Fairhurst, 2002).

Cualquier alternativa de aprovechamiento de la paja de arroz, debe ser viable económicamente en especial para los agricultores. La retirada de la paja pasa inevitablemente por establecer y favorecer la presencia de industrias capaces de utilizar la paja y generar las utilidades que permitan compensar el costo de su retirada, superiores a las opciones de incorporarla, o quemarla (Abril, et al., 2009).

Investigaciones realizadas por profesores de la Universidad de Sancti Spíritus en la empresa agroindustrial de granos Sur del Jíbaro demostró que la paja de arroz y el residuo del proceso de secado como única fuente de sustrato, tienen un significativo potencial de biogás, que alcanza un valor máximo de hasta $0,5 \mathrm{~m}^{3} \mathrm{~kg} \mathrm{SV}-1$ en termofílico. (Contreras, et al., 2012)

Son muchas las opiniones encontradas sobre el uso de la paja de arroz con respecto a su retirada o no del campo después de la cosecha, por una parte está la tendencia medioambientalista basada en los gases contaminantes que esta emite con una alta repercusión para el medio ambiente. Por otra parte la tendencia agronomista referida a los aportes que pudiera tener la incorporación de la paja al suelo y su aprovechamiento por el cultivo.

Consideramos que ambas tendencias tienen fe de causas por lo que la solución estaría en constatar cuales son específicamente las carencias que provocaría la retirada de la paja del campo y buscar alternativas de solución para reponer estas necesidades. 


\section{Materiales y métodos}

El trabajo fue realizado tomando en consideración las producciones de paja de la Empresa Agroindustrial de granos Sur del Jíbaro ubicada en la provincia de Sancti Spíritus, Cuba. Para el mismo se hizo un diagnóstico de las potencialidades para la obtención de paja de arroz en cinco años a partir del 2008, las cuales pudieran ser utilizadas en la producción de biogás. El diagnóstico incluyó los principales destinos que se le da en la actualidad a esa paja para lo cual se revisaron diferentes bases de datos de la empresa así como entrevistas con especialistas de la misma.

La empresa tiene una extensión de 83875 ha, de las cuales 27217 ha, se dedican al cultivo del arroz, 20130 ha a la ganadería y 2050 ha a los cultivos varios. El resto es superficie no agrícola, entre ellas canales de riego, viales e instalaciones. Los principales macizos arroceros están ubicados en tres regiones fundamentales. El primer macizo de arroz se ubica al sureste de los poblados de Mapo y Natividad, el segundo se extiende desde el poblado de Peralejo hasta El Jíbaro, por la parte sur de los mismos y llega aproximadamente hasta el litoral costero, mientras el tercero se encuentra ubicado al suroeste del poblado de Las Nuevas (Contrera, 2013)

Para el calculo de las cantidades de paja de arroz generadas por la Empresa se tomó como referencia los experimentos realizados por García, 2010 el cual utilizó un muestreo probabilístico intencional. Con este valor se pudo determinar un valor aproximado de la cantidad de paja seca que se produce por unidad de área durante la cosecha de arroz. El resultado obtenido se comparó con datos encontrados en la bibliografía y se pudo comprobar la similitud de los mismos.

Una vez obtenido el índice de la generación de este desecho, se le aplicó al área cosechada en los años desde 2008 hasta 2012, obteniéndose así las cantidades de paja de arroz producidas cada año.

\section{Resultados y discusión}

Después de aplicados los instrumentos para el diagnóstico pudimos encontrar que no existe en la empresa ninguna información relacionada con la cantidad de residuos que se produce en el proceso de recolección del grano. Si se pudo conocer que la misma varía en dependencia de varios factores como son, la variedad de arroz que se haya sembrado, la población del campo en cuestión, la altura a que se produce el corte de la planta entre otras. Al no encontrar dichos datos, se tomaron como referencia los índices obtenidos por García en el 2010 de 4.86 toneladas de paja por hectáreas de arroz sembradas lo cual fue comparado con otros experimentos hechos por otros autores por ejemplo según California Straw Building Association (CASBA) (2008) la producción de paja de arroz en California es de 5.43 toneladas por hectárea lo cual se asemeja a lo obtenido en Cuba en el 2010. 
Utilizando el índice antes mencionado se pudo calcular la cantidad aproximada de paja de arroz producida por la entidad en cinco años a partir de 2008 hasta el 2012. La cual se muestra en el siguiente gráfico:

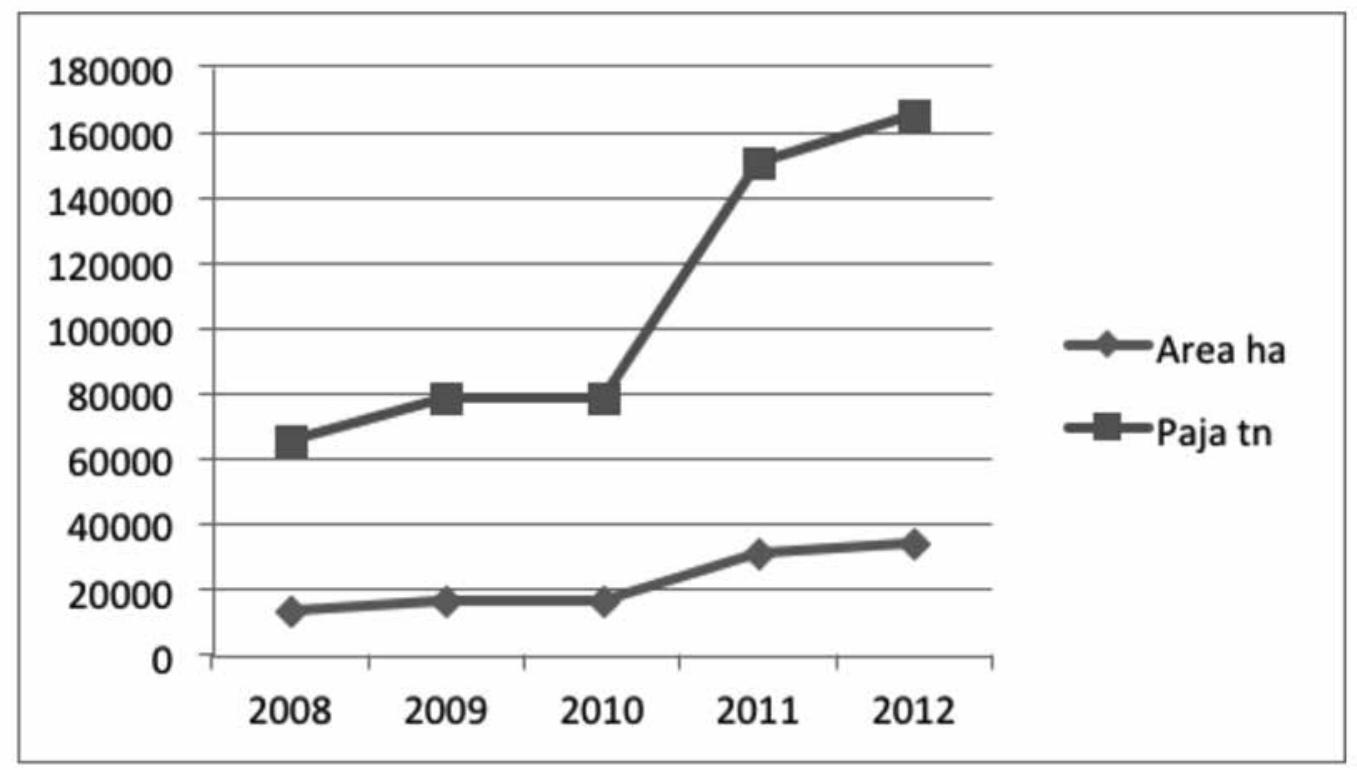

Se pudo comprobar además que los restos de la cosecha (paja de arroz), no cuentan actualmente con una disposición final ambientalmente adecuada ni se encuentran contabilizadas las cantidades según los destinos. Pequeñas cantidades no contabilizadas se emplean como alimento para el ganado en épocas de sequía, el resto queda depositada en el suelo y son posteriormente quemadas a cielo abierto cuando se va a preparar la tierra para la nueva cosecha (García, 2010; Linares y Meneses, 2011) y unas cantidades importantes son incorporadas al suelo durante el fangueo según explican varios especialistas conocedores del tema.

Tomando en consideración estos altos volúmenes de paja que se vienen incrementando a medida que crecen las áreas productivas y que por regla la mayor parte de estos residuos quedan en el campo es que se hace más viable la propuesta que se viene trabajando hace varios años por profesores de la Universidad de Sancti Spíritus para la instalación de plantas de biodigestores para el tratamiento de los antes mencionado volúmenes de paja que se generan cada año.

La utilización de biodigestores ofrece grandes ventajas para el tratamiento de los desechos orgánicos de las explotaciones agropecuarias, pues además de disminuir la carga contaminante de las mismas, extrae gran parte de la energía contenida en el material sin afectar (o inclusive mejorando) su valor fertilizante y controlando de manera considerable los malos olores. 
Según el rendimiento de biogás experimental, es posible obtener una producción de 108675 m3. A partir de la producción diaria de biogás esperada y los índices de generación de energía eléctrica y térmica empleados, se puede estimar una producción de energía de $242344 \mathrm{kWhd}-1$ eléctricos para una potencia de $10 \mathrm{MW}$ y una disponibilidad de energía térmica de $360799 \mathrm{kWhd}-1$ para una potencia de $15 \mathrm{MW}$, lo que posibilitaría a la empresa el autoabastecimiento de energía eléctrica y la sustitución del diesel que consume en la etapa industrial de secado. (Contreras, 2013)

Otro de las significativas ventajas que esto ofrecería es la disminución considerable de los gases contaminantes que hoy produce la descomposición o quema de esta paja en el campo.

Según Contreras, 2013, sobre la base de los datos de producción de la Empresa Agroindustrial de Granos "Sur del Jíbaro" durante el año 2011, se obtuvo que se hubieran dejado de emitir a la atmósfera $6041438 \mathrm{t}$ de $\mathrm{CO}_{2}$ equivalente si la paja de arroz hubiese sido retirada del campo y tratada por vía anaerobia. Esto hubiera significado el o,2\% de las emisiones totales del país y el 3,2\% de las emisiones del sector energético en ese propio año.

En cambio la incorporación de la paja de arroz al suelo, con el tiempo, puede reducir el rendimiento de la cosecha por el deterioro de sus condiciones e influir en el incremento de las enfermedades foliares (Zhang R. y Zhang Z., 1999), además de las emisiones de metano que se emiten a la atmósfera debido a la degradación natural (Watanable, et al., 1995).

Aún considerando los criterios de autores como Datnoff, et al., 1997, quien dice que el retiro de la paja de arroz del suelo, implicaría la extracción de nutrientes, fundamentalmente $\mathrm{N}, \mathrm{P}$ y $\mathrm{K}$, haciendo necesaria su reincorporación con la fertilización y que además se retira silicio en forma de dióxido de silicio amorfo; creemos que es factible la propuesta de la extracción de la paja para aprovecharla en la obtención de energía.

Según especialistas que han trabajado el tema, el digestato producto de la conversión de la paja de arroz pudiera ser una fuente importante de nutrientes que podría satisfacer las necesidades de cultivo una vez extraída la paja del campo, por lo que sería prudente caracterizar este digestato y experimentar en diferentes dosis para comprobar su efectividad.

\section{Conclusiones}

1. Los grandes volúmenes de paja que se generan después de la cosecha del arroz en la Empresa agroindustrial de granos Sur del Jíbaro emiten altas concentraciones de gases contaminantes que en la actualidad no tienen un manejo medioambiental adecuado. 
2. Existen potencialidades en dicha entidad para generar la energía eléctrica que satisfaga sus demandas en el proceso industrial de granos a través de la utilización de dicha paja en biodigestores.

3. Existen potencialidades para la producción de un digestato capaz de aportar los elementos nutritivos que demande el cultivo una vez extraída la paja después de la cosecha.

\section{Recomendaciones}

1. Realizar trabajos experimentales para evaluar la efectividad del digestato obtenido en el proceso de bioconversión de la paja de arroz al ser utilizado como biofertilizante.

\section{Lista de referencias}

Abril, D. Navarro, E y Abril, A. (2009). La paja de arroz. Consecuencias de su manejo y alternativas de aprovechamiento.

California Straw Building Association (CASBA). (2008). California rice Straw production FAQ. [en línea]. Disponible en: http://www ricestrawmarket.org.

Contreras, L. M. (2013). Digestión anaerobia de residuos de la agroindustria arrocera cubana para la producción de biogás. Tesis en opción al grado científico de Doctor en Ciencias Técnicas.

Contreras, L .M., Pereda, 1., Romero, O. (2012). Aprovechamiento energético de residuos arroceros por bio-conversión caso de estudio Cuba.

Datnoff, L. E., Deren, C. W. \& Snyder, G. H. (1997). Silicon fertilization for disease managment in Florida. In:Crop Protection 16, No. 6. pp. 525-531.

Dockery, D. W., Pope, A. C., Xu X., Spengler, J. D., Ware, J. H., Fay, M. E., Ferris, Jr. B. G. \& Speizer, F. E. (1993). An association between air pollution and mortality in six U. S. Cities. In: The New England Journal of Medicine, No. 329. pp.1753-1759.

Doberman, A. \&Fairhurst, T. H. (2002). Rice straw management. En: Better Crops International, Vol. 12. Special Supplement.

Fitzgerald, G. J., Scow, K. M. \& Hill, J. E. (200o). Fallow season straw and water management effects on methane emissions in California rice. In: Global Biogeochemical Cycles, No. 14. pp. 767-776. 
Gadde, B., Christoph, M. y Reiner, W. (2009). Rice straw as a renewable energy source in India, Thailand, and the Philippines: Overall potential and limitations for energy contribution and greenhouse gas mitigation. Biomass and Bioenergy, 33, 1532-1546.

García, A. (2010). Mejoramiento de la eficiencia energética y económica del CAI Arrocero Sur del Jíbaro mediante estudio de potenciales de ahorro por aprovechamiento energético de residuos. Tesis en opción al grado de Master. Maestría de Eficiencia Energética. Centro Universitario “José Martí Pérez”. Sancti Spíritus, Cuba.

Kaufman, Y. J., Tanre, D. \& Boucher, O. (2002). A satellite view of aerosols in the climate system. In: Nature, No. 419. pp. 215-223.

Linares, O. y Menéses, C. (2011). Comunicación personal con el Director y subdirector de Industria: Complejo Agroindustrial Arrocero CAI "Sur del Jíbaro". Sancti Spíritus, diciembre 2011.

Navarro, E. (2008). La biomasa de la Albufera, aprovechamiento y corrección de impactos. Valencia: Ed. Aleta Ediciones. Colección Aleta Investigación. 156 p. 